\title{
Role of the PI3K/AKT signalling pathway in apoptotic cell death in the cerebral cortex of streptozotocin-induced diabetic rats
}

\author{
YAN MENG, WEIWEI WANG, JINSONG KANG, XINXUE WANG and LIANKUN SUN \\ Department of Pathophysiology, Key Laboratory of Pathobiology, Ministry of Education, \\ College of Basic Medicine, Jilin University, Changchun, Jilin 130021, P.R. China
}

Received September 1, 2015; Accepted January 26, 2017

DOI: $10.3892 /$ etm.2017.4259

\begin{abstract}
Diabetes mellitus is associated with cognitive dysfunction. Numerous previous studies have shown that type 1 diabetes-induced hyperglycaemia causes structural brain damage, such as a decrease in whole-brain grey matter. The impact of diabetes mellitus on the cerebral cortex is poorly understood and requires further clarification. In the present study, diabetes was induced via an intraperitoneal injection of streptozotocin $(50 \mathrm{mg} / \mathrm{kg})$. Hematoxylin and eosin (H\&E) staining was performed to detect the morphological changes in the cerebral cortex, terminal deoxynucleotidyl transferase-mediated dUTP nick end labelling (TUNEL) staining was used to detect neuronal apoptosis and western blotting was performed to determine protein expression levels. Nine weeks after the induction of diabetes, the body weight was significantly lower and the blood glucose levels were significantly higher in the diabetic rats than in the control rats $(\mathrm{P}<0.05)$. H\&E staining revealed nuclear chromatin condensation and cytoplasmic shrinkage in the cerebral cortex of the diabetic rats and TUNEL staining further indicated apoptotic changes in the cerebral cortex of the diabetic rats. The ratio of B-cell lymphoma 2 (Bcl-2) -associated X protein/Bcl-2 and the expression of cytochrome $c$ and activated caspase-3 (cleaved caspase-3) were significantly increased, whereas the ratio of phosphorylated AKT/AKT was significantly decreased in the diabetic rats compared with that in the control rats $(\mathrm{P}<0.05)$. Taken together, these results suggested that diabetes mellitus
\end{abstract}

Correspondence to: Professor Liankun Sun, Department of Pathophysiology, Key Laboratory of Pathobiology, Ministry of Education, College of Basic Medicine, Jilin University, 126 Xinmin Street, Changchun, Jilin 130021, P.R. China

E-mail:sunlk@jlu.edu.cn

Abbreviations: STZ, streptozotocin; H\&E, haematoxylin and eosin; TUNEL, terminal deoxynucleotidyl transferase-mediated dUTP nick end labelling; DM, diabetes mellitus; PKB, protein kinase B; phos-AKT, phosphorylated AKT

Key words: diabetes mellitus, streptozotocin, cerebral cortex, apoptosis, AKT, Bax, Bcl-2 may induce neuronal apoptosis in the cerebral cortex by downregulating AKT phosphorylation.

\section{Introduction}

Diabetic complications are known to be able to affect the central nervous system, a condition that is referred to as diabetic encephalopathy. The effects of diabetes mellitus on the central nervous system have received a large amount of attention from investigators. In diabetic encephalopathy, which was initially proposed by Nielsen and Lundbaek, disrupted glucose metabolism in diabetes mellitus causes pathological changes in cerebrovascular and brain structures, which leads to damage of the central nervous system. Diabetic encephalopathy is clinically characterised by moderate cognitive deficits, which manifest as impairments in learning and memory and difficulties regarding space-time awareness (1-3). As the clinical manifestations of diabetic encephalopathy are non-specific, a clear definition and diagnostic criteria for this functional impairment remain to be clarified $(4,5)$. Perantie et al (6) found that exposure to hyperglycaemia was associated with an accentuated decrease in whole-brain grey matter over a 2-year period in adolescents with type 1 diabetes mellitus. Clinical studies have demonstrated that atrophy of the cerebral cortex and neuronal degeneration are consequences of the cognitive dysfunction caused by diabetes mellitus, although the histopathological changes found in the brain of diabetes mellitus patients have yet to be characterized $(7,8)$. Experimental studies further suggested that apoptosis is a potential mechanism underlying neuronal death in diabetes mellitus. Diabetes mellitus-induced brain injury (e.g., memory and cognitive deficits) is at least partially mediated by neuronal apoptosis $(9,10)$.

The phosphatidylinositol 3-kinase (PI3K) signalling pathway promotes cell survival and has been reported to participate in apoptosis in the central nervous system. AKT, a serine/threonine protein kinase that is also known as protein kinase $\mathrm{B}(\mathrm{PKB})$, is the primary protein effector downstream of the PI3K signalling pathway. AKT has an important role in glucose metabolism by regulating the biological function of insulin. The effect of AKT on glucose metabolism regulation upon insulin stimulation primarily relies on the following two mechanisms: AKT induces the translocation of glucose transporter type 4 to the plasma membrane, thereby mediating 
glucose uptake (11-13); furthermore, AKT phosphorylates and inhibits the activity of glycogen synthase kinase 3 , which increases the activity of glycogen synthase and promotes glycogen synthesis $(14,15)$. In addition, AKT is among the most important apoptosis-inhibiting proteins. Allelic mutations of AKT decrease the pro-survival effect of growth factors and other nutrients, whereas overexpression of wild-type or activated AKT rescues cells from apoptosis induced by various types of stress $(16,17)$.

A vast number of experimental studies have shown that the AKT signalling pathway is involved in the pathophysiological processes of diabetes mellitus and its complications $(18,19)$. Malfunction of the AKT signalling pathway during hyperglycaemia may increase myocardial cell apoptosis, which is accompanied by an increase in the release of cytochrome $c$ from mitochondria and an enhancement of caspase-3 activity (20). Diabetes mellitus induces neuronal apoptosis in the hippocampus and decreases the levels of phosphorylated (phos-) AKT. However, luteolin inhibits the release of cytochrome $c$ and the activation of caspase-3 via the upregulation of AKT phosphorylation, thereby inhibiting the apoptosis of hippocampal neurons in diabetes mellitus (21).

Diabetes mellitus is associated with cognitive dysfunction. The cerebral cortex has an important role in cognitive function but the mechanism by which diabetes mellitus induces the formation of cerebral cortical lesions has remained elusive. Thus, the present study investigated whether diabetes mellitus induces neuronal apoptosis in the cerebral cortex and evaluated the role of AKT in the mechanism underlying cerebral cortical injury in diabetes.

\section{Materials and methods}

Animals. A total of 18 male 8 -week-old Wistar rats $(\mathrm{n}=9$ per group, weight, 180-220 g) were purchased from the Laboratory Animal Center of the College of Basic Medicine of Jilin University [Changchun, China; license no. SCXK (Ji) 2007-0003]. The animals were housed in standard laboratory cages at room temperature with a 12-h light/dark cycle and were provided free access to food and water throughout the study period. All experimental protocols and procedures were approved by the Animal Care and Ethics Committee of Jilin University (Changchun, China). All experiments were performed in accordance with the National Institutes of Health Guide for the Care and Use of Laboratory Animals. The experimental animals were randomly divided into two groups, the control group $(\mathrm{CON}, \mathrm{n}=9)$ and the diabetes mellitus group (DM, n=9).

Drugs and antibodies. Streptozotocin (STZ) was from Sigma-Aldrich (Merck KGaA, Darmstadt, Germany). The primary antibodies used in the present study, including antibodies against $\beta$-actin (sc-47778), B-cell lymphoma 2 (Bcl-2; sc-492), Bcl-2-associated X protein (Bax; sc-493), cytochrome c (sc-13156), caspase-3 (sc-136219), AKT (sc-55523) and phos-AKT (sc-7985), were purchased from Santa Cruz Biotechnology, Inc. (Dallas, TX, USA). Corresponding horseradish peroxidase-labelled secondary antibodies ( $\mathrm{SH}-0012$ and SH-0032) were purchased from Ding Guo Changsheng Biotechnology Co., Ltd. (Beijing, China).
Rat model of diabetes mellitus. Wistar rats were adaptively fed for one week and then fasted for $12 \mathrm{~h}$ prior to establishment of the diabetes mellitus model. The rat model of diabetes mellitus was induced via a single injection of $50 \mathrm{mg} / \mathrm{kg} \mathrm{STZ}$, which was freshly dissolved in $100 \mathrm{mM}$ sodium citrate buffer (pH 4.2, $0.1 \mathrm{mM}$ ), into the left lower abdominal cavity. The rats in the control group were treated with sodium citrate buffer alone. After the injection of STZ, the rats were fed adequate amounts of food and water. Diabetes was verified on the third day after the STZ injection by evaluating the blood glucose levels using a blood glucose monitor (ACCU-CHEK Integra, Roche Diagnostics GmbH, Mannheim, Germany). The rats that displayed a blood glucose level of at least $16.7 \mathrm{mM}$ were considered to be diabetic. Once the diabetes model was established, the rats were reared routinely, their body weight was measured weekly and their blood glucose levels were measured every fortnight for a total of 9 weeks. On the ninth week after STZ injection, the rats were anaesthetised with sodium pentobarbital ( $80 \mathrm{mg} / \mathrm{kg}$, intraperitoneally; Beijing Propbs Biotechnology, Co., Ltd., Beijing, China) and sacrificed for subsequent experimental use.

Haematoxylin and eosin $(H \& E)$ staining. The rats were anaesthetised and fixed by perfusing with 200-300 ml fixative (4\% paraformaldehyde in 0.1 M PBS, pH 7.4) through the left ventricle of the heart. The brain tissues were immediately collected and coronal sections of the brain were fixed in $4 \%$ paraformaldehyde at $4^{\circ} \mathrm{C}$ for $24 \mathrm{~h}$. The brains were processed via dehydration using a graded ethanol series, cleared with xylene and routinely embedded in paraffin. Serial coronal sections $(10 \mu \mathrm{m})$ of the forebrain were cut, deparaffinised, rehydrated and then stained with H\&E. Finally, the morphology of the cerebral cortex was observed via light microscopy (Olympus, Tokyo, Japan).

Terminal deoxynucleotidyl transferase-mediated dUTP nick end labelling (TUNEL) staining. The TUNEL staining method was applied to detect apoptosis in the rat cerebral cortex according to the manufacturer's instructions (cat. no. 11684795910; Roche, Basel, Switzerland). Following TUNEL staining, the sections were examined under a light microscope (Olympus) to observe apoptotic neurons in the cortex. The cells that were positively stained with TUNEL showed a dark brown or yellow colour concentrated in the nucleus under the light microscope and were considered to be apoptotic. The numbers of neurons and TUNEL-positive cells in each section were counted. Five full representative fields of view (magnification, x400) were randomly selected from each section to quantify the neurons and TUNEL-positive cells, and the apoptotic rate was calculated using the standard formula.

Western blot analysis. After the rats were decapitated, the cerebral cortex was segregated and the pieces were placed in liquid nitrogen for rapid freezing and homogenisation. In brief, a homogenate was prepared from $100 \mathrm{mg}$ frozen cerebral cortical tissue in $0.5 \mathrm{ml}$ homogenisation buffer containing $25 \mathrm{mM}$ Tris- $\mathrm{HCl}$ (pH 7.6), $150 \mathrm{mM} \mathrm{NaCl}, 1 \%$ Nonidet P-40, $1 \%$ sodium deoxycholate, $0.1 \%$ sodium dodecyl sulphate (SDS), $1 \mu \mathrm{g} / \mathrm{ml}$ phenylmethanesulphonyl fluoride, $1 \mu \mathrm{g} / \mathrm{ml}$ 
leupeptin, $1 \mu \mathrm{g} / \mathrm{ml}$ aprotinin, $1 \mu \mathrm{g} / \mathrm{ml}$ pepstatin and $1 \mathrm{mM}$ dithiothreitol. After sonication on ice, the homogenates were centrifuged for $10 \mathrm{~min}$ at 1,200 x $\mathrm{g}$. The supernatants were further centrifuged for $5 \mathrm{~min}$ at $12,000 \times \mathrm{g}$ to obtain total protein samples. The protein concentration was determined using a protein assay kit (Bio-Rad Laboratories, Inc., Hercules, CA, USA). Western blotting was performed using standard techniques. The lysate protein samples $(60 \mu \mathrm{g})$ were separated via $12 \%(\mathrm{w} / \mathrm{v})$ SDS-PAGE and transferred onto polyvinylidene difluoride membranes. The membranes were blocked in PBST buffer ( $\mathrm{pH}$ 7.6) containing $10 \mathrm{mM}$ Tris- $\mathrm{HCl}, 5 \%(\mathrm{w} / \mathrm{v})$ skimmed milk, $100 \mathrm{mM} \mathrm{NaCl}$ and $0.1 \%$ (v/v) Tween-20 for $1.5 \mathrm{~h}$ at room temperature, followed by incubation with the primary antibodies at 1:1,000 dilution overnight at $4^{\circ} \mathrm{C}$. The membranes were then incubated with the corresponding horseradish peroxidase-conjugated secondary antibodies (Ding Guo, Beijing, China) at a 1:2,000 dilution for $1 \mathrm{~h}$ at room temperature. The immunoreactive bands were visualised using diaminobenzidine (Sigma-Aldrich; Merck KGaA). The primary antibodies used in the present study were mouse monoclonal antibodies against $\beta$-actin, Bax and cytochrome $c$ and rabbit polyclonal antibodies against Bcl-2, caspase-3, AKT and phos-AKT all of which were purchased from Santa Cruz Biotechnology, Inc. $\beta$-actin was used as an internal control. The protein levels were quantified using Quantity One software version 4.62 (Bio-Rad Laboratories, Inc.) by calculating the band intensity (area $\mathrm{x}$ absorbance) normalised to that of the internal control in each group.

Statistical analysis. All values are expressed as the mean \pm standard deviation. Statistical analysis was performed using SPSS 13.0 software (SPSS, Inc., Chicago, IL, USA). One-way analysis of variance followed by Dunnett's t-test was used for comparison between the groups. $\mathrm{P}<0.05$ was considered to indicate a statistically significant difference.

\section{Results}

Changes in the blood glucose levels and body weight of STZ-induced diabetic rats. STZ injection resulted in diabetic syndrome, which was verified by the presence of polydipsia, polyuria and hyperglycaemia in the diabetic animals. The fasting blood glucose levels and the body weight of the rats in the two groups were similar prior to the experiment. The blood glucose levels of the rats in the DM group were significantly increased $(>16.7 \mathrm{mM} ; \mathrm{P}<0.01$ vs. the $\mathrm{CON}$ group at the same week) and remained in a hyperglycaemic state throughout the 9 weeks after STZ injection. However, no apparent change in the blood glucose levels was detected in the CON group (Fig. 1A). The body weight of the rats in each group increased over time throughout the experimental period. At the end of the ninth week after STZ injection, the body weight of the diabetic rats was significantly lower than that of the control rats (Fig. 1B). These results confirmed the validity of the rat model of type 1 diabetes.

Changes in cerebral cortex morphology in the STZ-induced diabetic rats. Based on $\mathrm{H} \& \mathrm{E}$ staining analysis, a normal distribution of neurons was observed in the cerebral cortex of the CON group, which included pyramidal cells with elliptic
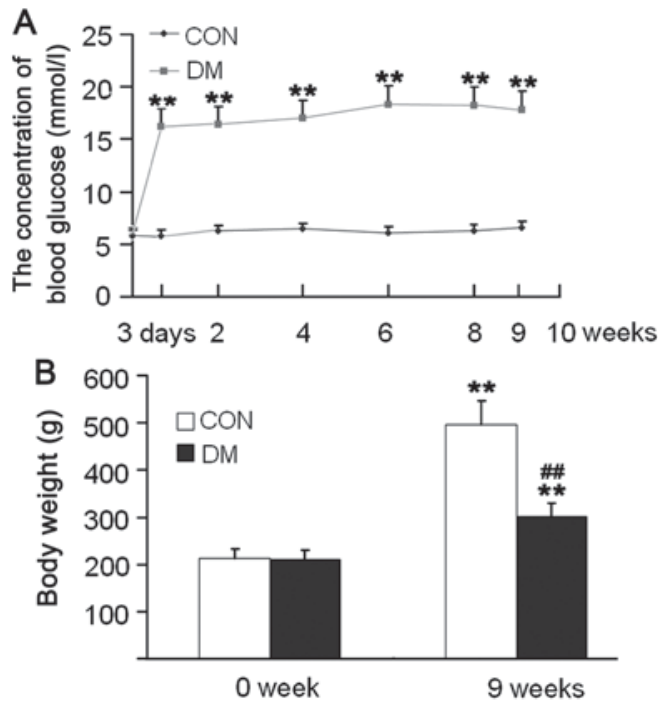

Figure 1. (A) Blood glucose concentration and (B) body weight over several weeks after streptozotocin injection. ${ }^{* *} \mathrm{P}<0.01$ compared with the 1 -week group and ${ }^{\# \#} \mathrm{P}<0.01$ compared with the $\mathrm{CON}$ group ( $\mathrm{n}=9$ per group). $\mathrm{CON}$, control group; DM, diabetic group.

neuronal bodies, small neurites, rounded nuclei, well-defined nucleoli and a basophilic cytoplasm. However, the organisational structure in the cerebral cortex of the DM group was altered. It was clearly observed that in the DM group, the number of neurons exhibiting a normal morphology was reduced, as most neurons appeared as shrunken dark cells and were much more frequently polygonal or fan-shaped. Cells with nucleoplasm condensation, pyknosis and an ambiguous nuclear membrane structure, all of which are suggestive of apoptosis, were also observed (Fig. 2A). The results of H\&E staining indicated that diabetes mellitus induced neuronal injury in the cerebral cortex.

Apoptotic changes in the cerebral cortex of the STZ-induced diabetic rats. Apoptotic cells were detected via TUNEL staining (Fig. 2B). The TUNEL-positive cells were considered as apoptotic cells. In the control rats, few TUNEL-positive cells were detected in the cerebral cortex, and the TUNEL-negative cells, indicating normal cells, displayed deeply stained nuclei, lightly stained cytoplasm and maintained cell membrane integrity as well as a rounded cell structure, in contrast to the intensely stained buff-coloured and shrunken nuclei and strongly stained cell membranes. In the DM group, the number of TUNEL-positive cells was significantly increased. The apoptotic rate in the DM group was significantly higher than that in the CON group $(\mathrm{P}<0.05)$ (Fig. 2C).

Expression of the apoptosis-associated proteins Bax, Bcl-2, cytochrome $c$ and caspase-3 in the cerebral cortex of the STZ-induced diabetic rats. To further confirm that diabetes mellitus induced apoptosis in the cerebral cortex, western blotting was performed to detect apoptosis-associated protein expression. As shown in Fig. 3, the expression level of Bax was upregulated and the expression of Bcl-2 was downregulated in the DM group compared with that in the $\mathrm{CON}$ group. The ratio of Bax/Bcl-2 $(\mathrm{P}<0.01)$ and the expression of cytochrome $c$ $(\mathrm{P}<0.05)$ and cleaved caspase-3 $(\mathrm{P}<0.01)$ was significantly 


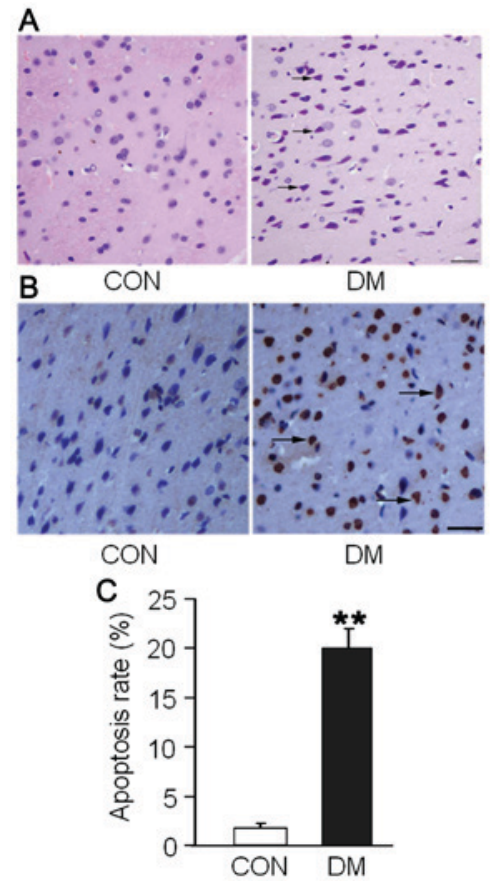

Figure 2. Morphological and apoptotic changes in the rat cerebral cortex at 8 weeks after STZ injection. (A) Representative hematoxylin and eosin staining. Black arrows indicate representative damaged cells. (B) Photomicrographs of TUNEL-positive neurons in the cerebral cortex of the various groups. Black arrows indicate representative TUNEL-positive cells. Light microscopy, $\mathrm{x} 400$; scale bar, $25 \mu \mathrm{m}$. (C) Quantitative analysis of the apoptotic cells in the cerebral cortex of the STZ-induced diabetic rats. Values are presented as the mean \pm standard deviation ( $n=3$ per group). ${ }^{* *} \mathrm{P}<0.01$ compared with the CON group. STZ, streptozotocin; CON, control group; DM, diabetic group; TUNEL, terminal deoxynucleotidyl transferase-mediated dUTP nick end labelling.

increased, but the expression level of caspase-3 precursor $(\mathrm{P}<0.05)$ was significantly decreased in the cerebral cortex of the DM group compared with that in the CON group. The above findings indicated that in the cerebral cortex, diabetes mellitus induced apoptosis via the mitochondrial apoptotic pathway.

Expression of AKT and phos-AKT in the cerebral cortex of STZ-induced diabetic rats. To determine whether apoptosis in the cerebral cortex of the STZ-induced diabetic rats is mediated via the PI3K pathway, western blot analysis was performed to detect the phosphorylation of AKT, the primary protein effector downstream of the PI3K signalling pathway. As shown in Fig. 4, the level of phos-AKT, but not AKT, was significantly decreased in the cerebral cortex of the DM group compared with that in the CON group $(\mathrm{P}<0.01)$. This finding indicated that inhibition of the PI3K pathway is involved in the mechanism underlying apoptosis in the cerebral cortex of STZ-induced diabetic rats.

\section{Discussion}

Hyperglycaemia and body weight loss are the most important characteristic changes caused by diabetes mellitus. In the present study, after a single intraperitoneal injection of STZ, the blood glucose levels of rats were significantly increased $(>16.7 \mathrm{mM})$, and this hyperglycaemic state was maintained for
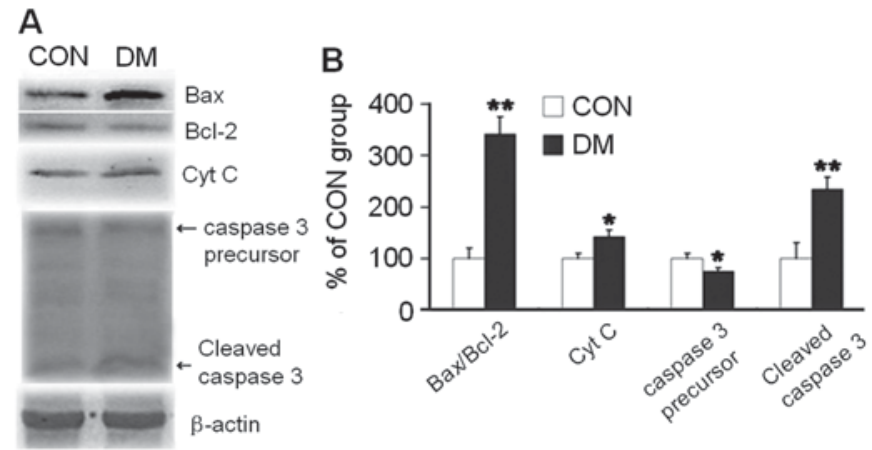

Figure 3. Western blot analysis of the expression level of apoptosis-related proteins in the rat cerebral cortex at 8 weeks after streptozotocin injection. (A) Western blot for Bax, Bcl-2, Cyt $C$ and caspase-3 in the cerebral cortex. (B) Quantification of the expression levels of these proteins in the cerebral cortex. The relative expression levels of these proteins were normalised to those of $\beta$-actin and are presented as the ratio of the values of the DM group to those of the CON group. Values are presented as the mean \pm standard deviation ( $\mathrm{n}=3$ per group). ${ }^{*} \mathrm{P}<0.05$ and ${ }^{* *} \mathrm{P}<0.01$ compared with the CON group. CON, control group; DM, diabetic group. Bcl-2, B-cell lymphoma 2; Bax, Bcl-2-associated X protein; Cyt $C$, cytochrome $c$.
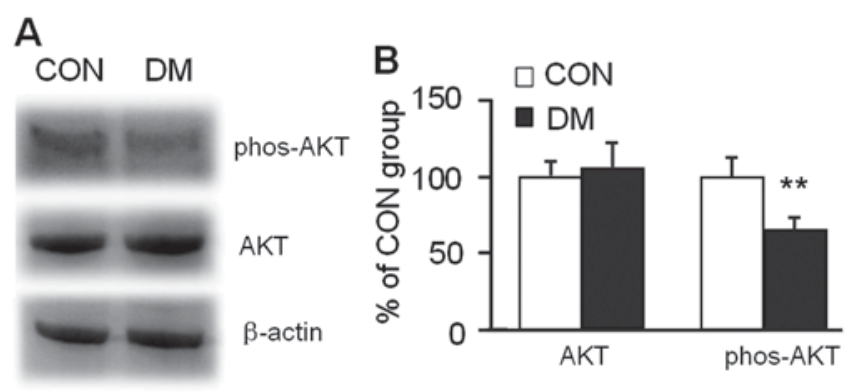

Figure 4. Western blot analysis to determine the expression of AKT and phos-AKT in the rat cerebral cortex at 8 weeks after streptozotocin injection. (A) Western blots for AKT and phos-AKT in the cortex. (B) Quantification of total AKT and phos-AKT in the cortex. The relative expression levels of these proteins were normalised to those of $\beta$-actin and are presented as the ratio of the values of the DM group to those of the CON group. Values are expressed as the mean \pm standard deviation ( $\mathrm{n}=3$ per group). ${ }^{* *} \mathrm{P}<0.01$ compared with the CON group. CON, control group; DM, diabetic group; phos-AKT, phosphorylated AKT.

9 weeks. After 9 weeks, the body weight in the DM group was less than that in the CON group, indicating that the rat model of 1 type diabetes mellitus was successfully constructed. To determine whether the cerebral cortex of the diabetic rats was injured, H\&E staining was performed. Numerous characteristically eosinophilic neurons were observed in the cerebral cortex of the DM group and these neurons exhibited an altered organisational structure, confirming that diabetes mellitus induced cerebral cortical injury. Previous retrospective magnetic resonance imaging brain studies have associated differences in grey or white matter integrity with hypoglycaemia or hyperglycaemia in adolescents and adults with Type 1 DM (22-24). The studies suggested that exposure to glycaemic extremes may alter specific pathways or regions in the brain. It has been hypothesised that prolonged exposure to hyperglycaemia causes mitochondrial dysfunction and increased oxidative stress, leading to cell death in vulnerable brain regions $(25,26)$. The present study provided experimental evidence that STZ-induced type 1 diabetes mellitus produces 
persistent hyperglycaemia, which may result in cerebral cortical injury.

Neuronal apoptosis is a major form of neuronal death and has been regarded as a pathogenic factor of Alzheimer's disease (27) and vascular dementia (28). In the present study, the TUNEL staining results suggested that apoptosis may serve as the primary mechanism underlying injury to the cerebral cortex induced by diabetes mellitus.

Mitochondria are involved in apoptosis and serve as the regulatory centre of apoptosis $(29,30)$. Bcl-2 family proteins are the major regulators of the mitochondrial apoptotic pathway $(31,32)$. Cytochrome $c$ is the central mediator of the intrinsic, or mitochondrial, apoptotic pathway. The release of cytochrome $c$ from the mitochondria to the cytosol is common to a variety of apoptotic mechanisms $(33,34)$. Caspases are a family of specific cysteine proteases that are essential for apoptosis. Caspase- 3 acts as the critical effector or executor protease during the caspase cascade in apoptosis. Under normal conditions, caspase- 3 is expressed in the form of a zymogen; once activated by stress, caspase- 3 hydrolyses specific substrates, which results in apoptotic cell death $(35,36)$. Several studies have shown that the diabetic brain displays an increase in TUNEL-positive neurons, Bax expression and caspase-3 activity in the hippocampus, which was closely associated with a decrease in neuronal density and impaired performance in the Morris water maze test $(37,38)$. In the present study, to determine whether the mitochondrial pathway of apoptosis is involved in diabetes mellitus-induced injury to the cerebral cortex, the protein expression of Bax, Bcl-2, cytochrome $c$ and caspase- 3 in the cerebral cortex was examined. The western blot results showed that compared with the CON group, the expression level of Bax was upregulated and that of $\mathrm{Bcl}-2$ was downregulated in the DM group. The ratio of $\mathrm{Bax} / \mathrm{Bcl}-2$ $(\mathrm{P}<0.01)$, and the expression of cytochrome $c(\mathrm{P}<0.05)$ and cleaved caspase-3 $(\mathrm{P}<0.01)$ in the cerebral cortex of the DM group were significantly increased, but the expression levels of caspase- 3 precursor $(\mathrm{P}<0.05)$ were significantly decreased compared with those of the CON group. These results revealed that the mitochondrial pathway of apoptosis is activated in the rat cerebral cortex due to diabetes mellitus.

The PI3K/AKT signalling pathway is a key intracellular signal transduction pathway with a particularly important role in the regulation of apoptosis and survival. The pro-survival effect of AKT is primarily mediated by two mechanisms. First, AKT promotes cell survival via the direct phosphorylation of transcription factors, which results in the negative regulation of death-promoting genes (such as forkhead box protein $\mathrm{O} 1 \mathrm{a},-\mathrm{O} 3 \mathrm{a}$ and $-\mathrm{O} 4)$ or the positive regulation of pro-survival genes (such as nuclear factor- $\kappa$ B) $(39,40)$. Second, AKT promotes cell survival via direct phosphorylation of the primary regulators of the apoptosis cascade. Activated AKT phosphorylates the Serl36 site of Bcl-2-associated death promoter (BAD), leading to the dissociation of BAD from Bcl-2 and $\mathrm{Bcl}$ extra large protein, which effectively blocks BAD-induced apoptosis (41). AKT also phosphorylates the Serl84 site of Bax to inhibit its pro-apoptotic effect. In addition, AKT has an anti-apoptotic role by preventing the release of cytochrome $c$ and apoptosis-inducing factor from mitochondria $(42,43)$. Based on immunohistochemistry and western blot analysis, Nakajima et al (44) demonstrated that ischemic pre-conditioning resulted in the continuous phosphorylation and activation of AKT, which has an important role in the tolerance to cerebral ischemia. Ren et al (45) showed that the level of AKT activity in hippocampal tissue decreased after the establishment of diabetes mellitus, but that luteolin inhibited hippocampal neuronal apoptosis via the activation of AKT. To confirm whether diabetes mellitus also induces apoptosis in the cerebral cortex via the PI3K/AKT pathway, the expression levels of AKT and phos-AKT were detected via western blot in the present study. The results showed that the protein level of total AKT was not significantly changed, but that the level of phos-AKT was significantly decreased in the cerebral cortex due to diabetes mellitus. The results indicated that inhibition of the AKT pathway is involved in the apoptotic process induced by diabetes mellitus in the cerebral cortex.

In conclusion, the above histopathological and molecular biological results suggested that the PI3K/AKT pathway is involved in apoptosis in the cerebral cortex of diabetic rats. The inhibition of AKT phosphorylation may cause a reduction in the anti-apoptotic effect of AKT, which may increase the ratio of $\mathrm{Bax} / \mathrm{Bcl}-2$, the release of cytochrome $c$ and the activation of caspase-3, ultimately inducing apoptotic cell death in the cerebral cortex due to diabetes mellitus. Diabetes-induced memory and cognitive deficits may be partially attributed to the enhancement of apoptosis in the cerebral cortex. The present study enhanced the current understanding of the mechanisms involved in STZ-induced apoptosis in the cerebral cortex. Enhancing PI3K/AKT pathway activity may serve as a potential therapeutic strategy for diabetic encephalopathy.

\section{Acknowledgements}

The authors would like to express their gratitude to Dr Lihong Zhang at the Department of Pathology, College of Basic Medicine, Jilin University, for her excellent technical support. This work was supported by the National Natural Science Foundation of China (nos. 81272876, 81202552 and 81370240), the Jilin Provincial Research Foundation for International Science and Technology Cooperation Projects, China (no. 20150414026GH) and the Scientific Research Project of Health Department of Jilin Province (no. 2013z068).

\section{References}

1. Biessels GJ, van der Heide LP, Kamal A, Bleys RL and Gispen WH: Ageing and diabetes: Implications for brain function. Eur J Pharmacol 441: 1-14, 2002.

2. Reske-Nielsen E and Lundbaek K: Diabetic encephalopathy diffuse and focal lesions of the brain in long-term diabetes. Acta Neurol Scand Suppl 39 (Suppl4): S273-S290, 1963.

3. Sima AA: Encephalopathies: The emerging diabetic complications. Acta Diabetol 47: 279-293, 2010.

4. Mijnhout GS, Scheltens P, Diamant M, Biessels GJ, Wessels AM, Simsek S, Snoek FJ and Heine RJ: Diabetic encephalopathy: A concept in need of a definition. Diabetologia 49: 1447-1448, 2006.

5. Brands AM, Henselmans JM, de Haan EH and Biessels GJ: Diabetic encephalopathy: An underexposed complication of diabetes mellitus. Ned Tijdschr Geneeskd 147: 11-14, 2003 (In Dutch).

6. Perantie DC, Koller JM, Weaver PM, Lugar HM, Black KJ, White NH and Hershey T: Prospectively determined impact of type 1 diabetes on brain volume during development. Diabetes 60: 3006-3014, 2011. 
7. Kumar A, Haroon E, Darwin C, Pham D, Ajilore O, Rodriguez G and Mintz J: Gray matter prefrontal changes in type 2 diabetes detected using MRI. J Magn Reson Imaging 27: 14-19, 2008.

8. Korf ES, van Straaten EC, de Leeuw FE, van der Flier WM, Barkhof F, Pantoni L, Basile AM, Inzitari D, Erkinjuntti T, Wahlund LO, et al: Diabetes mellitus, hypertension and medial temporal lobe atrophy: The LADIS study. Diabet Med 24: 166-171, 2007.

9. Liu JP, Feng L, Zhang MH, Ma DY, Wang SY, Gu J, Fu Q, Qu R and Ma SP: Neuroprotective effect of liuwei dihuang decoction on cognition deficits of diabetic encephalopathy in streptozotocin-induced diabetic rat. J Ethnopharmacol 150 371-381, 2013.

10. Liu J, Feng L, Ma D, Zhang M, Gu J, Wang S, Fu Q, Song Y, Lan Z, Qu R and Ma S: Neuroprotective effect of paeonol on cognition deficits of diabetic encephalopathy in streptozotocin-induced diabetic rat. Neurosci Lett 549: 63-68, 2013.

11. Tsuchiya A, Kanno T and Nishizaki T: PI3 kinase directly phosphorylates Akt1/2 at Ser473/474 in the insulin signal transduction pathway. J Endocrinol 220: 49-59, 2013.

12. Sylow L, Kleinert M, Pehmøller C, Prats C, Chiu TT, Klip A, Richter EA and Jensen TE: Akt and Rac1 signaling are jointly required for insulin-stimulated glucose uptake in skeletal muscle and downregulated in insulin resistance. Cell Signal 26: 323-331, 2014.

13. Ji L, Zhang X, Liu W, Huang Q, Yang W, Fu F, Ma H, Su H, Wang H, Wang J, et al: AMPK-regulated and Akt-dependent enhancement of glucose uptake is essential in ischemic preconditioning-alleviated reperfusion injury. PLoS One 8: e69910, 2013

14. Leng Y, Wang Z, Tsai LK, Leeds P, Fessler EB, Wang J and Chuang DM: FGF-21, a novel metabolic regulator, has a robust neuroprotective role and is markedly elevated in neurons by mood stabilizers. Mol Psychiatry 20: 215-223, 2015.

15. Ishikawa M, Yoshida K, Okamura H, Ochiai K, Takamura H, Fujiwara $\mathrm{N}$ and Ozaki K: Oral porphyromonas gingivalis translocates to the liver and regulates hepatic glycogen synthesis through the Akt/GSK-3 $\beta$ signaling pathway. Biochim Biophys Acta 1832: 2035-2043, 2013.

16. Kauffmann-Zeh A, Rodriguez-Viciana P, Ulrich E, Gilbert C Coffer P, Downward J and Evan G: Suppression of c-Myc-induced apoptosis by ras signalling through $\mathrm{PI}(3) \mathrm{K}$ and PKB. Nature 385 544-548, 1997

17. Khwaja A, Rodriguez-Viciana P, Wennström S, Warne PH and Downward J: Matrix adhesion and ras transformation both activate a phosphoinositide 3-OH kinase and protein kinase B/Akt cellular survival pathway. EMBO J 16: 2783-2793, 1997.

18. Zdychová J and Komers R: Emerging role of AKT kinase/protein kinase B signaling in pathophysiology of diabetes and its complications. Physiol Res 54: 1-16, 2005.

19. Wang J, Yin H, Huang Y, Guo C, Xia C, Liu Q and Zhang L: Panax quinquefolius saponin of stem and leaf attenuates intermittent high glucose-induced oxidative stress injury in cultured human umbilical vein endothelial cells via PI3K/Akt/GSK-3 $\beta$ pathway. Evid Based Complement Alternat Med 2013: 196283, 2013.

20. Zhang W, Li R, Li J, Wang W, Tie R, Tian F, Liang X, Xing W, $\mathrm{He} \mathrm{Y}, \mathrm{Yu} \mathrm{L}$, et al: Alpha-linolenic acid exerts an endothelial protective effect against high glucose injury via PI3K/Akt pathway. PLoS One 8: e68489, 2013.

21. Whittington HJ, Harding I, Stephenson CI, Bell R, Hausenloy DJ, Mocanu MM and Yellon DM: Cardioprotection in the aging, diabetic heart: The loss of protective Akt signalling. Cardiovasc Res 99: 694-704, 2013.

22. Northam EA, Rankins D, Lin A, Wellard RM, Pell GS, Finch SJ, Werther GA and Cameron FJ: Central nervous system function in youth with type 1 diabetes 12 years after disease onset. Diabetes Care 32: 445-450, 2009.

23. Perantie DC, Wu J, Koller JM, Lim A, Warren SL, Black KJ, Sadler M, White NH and Hershey T: Regional brain volume differences associated with hyperglycemia and severe hypoglycemia in youth with type 1 diabetes. Diabetes Care 30: 2331-2337, 2007.

24. Ho MS, Weller NJ, Ives FJ, Carne CL, Murray K, Vanden Driesen RI, Nguyen TP, Robins PD, Bulsara M, Davis EA and Jones TW: Prevalence of structural central nervous system abnormalities in early-onset type 1 diabetes mellitus. J Pediatr 153 : 385-390, 2008.
25. Folli F, Guzzi V, Perego L, Coletta DK, Finzi G, Placidi C, La Rosa S, Capella C, Socci C, Lauro D, et al: Proteomics reveals novel oxidative and glycolytic mechanisms in type 1 diabetic patients' skin which are normalized by kidney-pancreas transplantation. PLoS One 5: e9923, 2010.

26. Rask-Madsen C and King GL: Mechanisms of disease: Endothelial dysfunction in insulin resistance and diabetes. Nat Clin Pract Endocrinol Metab 3: 46-56, 2007.

27. Cheng XL and Li MK: Effect of topiramate on apoptosis-related protein expression of hippocampus in model rats with Alzheimers disease. Eur Rev Med Pharmacol Sci 18: 761-768, 2014.

28. Zhang LM, Jiang CX and Liu DW: Hydrogen sulfide attenuates neuronal injury induced by vascular dementia via inhibiting apoptosis in rats. Neurochem Res 34: 1984-1992, 2009.

29. Altiparmak UE: Toxic optic neuropathies. Curr Opin Ophthalmol 24: 534-539, 2013

30. Sinha K, Das J, Pal PB and Sil PC: Oxidative stress: The mitochondria-dependent and mitochondria-independent pathways of apoptosis. Arch Toxicol 87: 1157-1180, 2013.

31. Topham CH and Taylor SS: Mitosis and apoptosis: How is the balance set? Curr Opin Cell Biol 25: 780-785, 2013.

32. Shamas-Din A, Kale J, Leber B and Andrews DW: Mechanisms of action of Bcl-2 family proteins. Cold Spring Harb Perspect Biol 5: a008714, 2013

33. Srinivasan S and Avadhani NG: Cytochrome c oxidase dysfunction in oxidative stress. Free Radic Biol Med 53: 1252-1263, 2012.

34. Kulikov AV, Shilov ES, Mufazalov IA, Gogvadze V, Nedospasov SA and Zhivotovsky B: Cytochrome c: The Achilles' heel in apoptosis. Cell Mol Life Sci 69: 1787-1797, 2012.

35. Smith MA and Schnellmann RG: Calpains, mitochondria and apoptosis. Cardiovasc Res 96: 32-37, 2012.

36. Van De Water TR, Lallemend F, Eshraghi AA, Ahsan S, He J, Guzman J, Polak M, Malgrange B, Lefebvre PP, Staecker H and Balkany TJ: Caspases, the enemy within and their role in oxidative stress-induced apoptosis of inner ear sensory cells. Otol Neurotol 25: 627-632, 2004.

37. Jafari Anarkooli I, Sankian M, Ahmadpour S, Varasteh AR and Haghir H: Evaluation of Bcl-2 family gene expression and caspase-3 activity in hippocampus STZ-induced diabetic rats. Exp Diabetes Res 2008: 638467,2008.

38. Guo YJ, Wang SH, Yuan Y, Li FF, Ye KP, Huang Y, Xia WQ and Zhou Y: Vulnerability for apoptosis in the hippocampal dentate gyrus of STZ-induced diabetic rats with cognitive impairment. J Endocrinol Invest 37: 87-96, 2014.

39. Peng C, Ma J, Gao X, Tian P, Li W and Zhang L: High glucose induced oxidative stress and apoptosis in cardiac microvascular endothelial cells are regulated by FoxO3a. PLoS One 8: e79739, 2013.

40. Romashkova JA and Makarov SS: NF-kappaB is a target of AKT in anti-apoptotic PDGF signalling. Nature 401: 86-90, 1999.

41. Song G, Ouyang G and Bao S: The activation of AKT/PKB signaling pathway and cell survival. J Cell Mol Med 9: 59-71, 2005.

42. Gibson EM, Henson ES, Haney N, Villanueva J and Gibson SB: Epidermal growth factor protects epithelial-derived cells from tumor necrosis factor-related apoptosis-inducing ligand-induced apoptosis by inhibiting cytochrome c release. Cancer Res 62: 488-496, 2002.

43. Kim EC, Yun BS, Ryoo IJ, Min JK, Won MH, Lee KS, Kim YM, Yoo I- and Kwon YG: Complestatin prevents apoptotic cell death: Inhibition of a mitochondrial caspase pathway through AKT/PKB activation. Biochem Biophys Res Commun 313: 193-204, 2004

44. Nakajima T, Iwabuchi S, Miyazaki H, Okuma Y, Kuwabara M, Nomura Y and Kawahara K: Preconditioning prevents ischemia-induced neuronal death through persistent Akt activation in the penumbra region of the rat brain. J Vet Med Sci 66: 521-527, 2004

45. Ren G, Kong J, Jia N and Shang X: Luteolin attenuates neuronal apoptosis in the hippocampi of diabetic encephalopathy rats. Neural Regen Res 8: 1071-1080, 2013. 